\title{
Elevated levels of soluble PD-L1 are associated with reduced recurrence in papillary thyroid cancer
}

\author{
Marra Jai Aghajani ${ }^{1,2}$, Tara Laurine Roberts ${ }^{1,2,3}$, Tao Yang ${ }^{2,4,5}$, Charles Eugenio McCafferty ${ }^{1,2}$, Nicole J Caixeiro ${ }^{1,2,6}$, \\ Paul DeSouza ${ }^{1,2,3}$ and Navin Niles ${ }^{1,2,7,8}$ \\ 'Ingham Institute for Applied Medical Research, Liverpool, New South Wales, Australia \\ ${ }^{2}$ School of Medicine, Western Sydney University, Campbelltown, New South Wales, Australia \\ ${ }^{3}$ South West Sydney Clinical School, UNSW Sydney, Sydney, Australia \\ ${ }^{4}$ Saint Vincent's Clinical School, UNSW Sydney, Sydney, Australia \\ ${ }^{5}$ SydPath, Saint Vincent's Hospital, Sydney, Australia \\ ${ }^{6}$ Centre for Oncology Education and Research Translation (CONCERT), Liverpool, New South Wales, Australia \\ ${ }^{7}$ Department of Head \& Neck Surgery, Liverpool Hospital, Liverpool, New South Wales, Australia \\ ${ }^{8}$ Department of Clinical Medicine, Faculty of Medicine and Health Sciences, Macquarie University, Sydney, Australia
}

Correspondence should be addressed to M J Aghajani: marra.aghajani1@gmail.com

\begin{abstract}
To date, no research evaluating the predictive capabilities of soluble programmed cell death-ligand 1 (SPD-L1) in thyroid cancer patients has been performed. We aimed to investigate the prognostic significance of SPD-L1 expression in papillary thyroid cancer (PTC) and to evaluate the association between SPD-L1 levels with tumoural PD-L1 expression and patient outcomes. Pre-treatment levels of serum and plasma SPD-L1 were measured by ELISA in 101 PTC patients. Tissue microarrays were stained with an antiPD-L1 antibody, clone SP263 (Ventana). The median serum SPD-L1 concentration in PTC patients was significantly higher compared to healthy controls $(P=0.028)$. An increased incidence of extrathyroidal extension was significantly associated with an elevated serum SPD-L1 level $(P=0.015)$. Patients with high serum SPD-L1 levels had significantly shorter median disease-free survival (DFS) as compared to those with low SPD-L1 levels $(P=0.011)$. Following multivariate analysis, serum SPD-L1 was the only statistically significant predictor for DFS. Patients with both positive serum and tumoural PD-L1 expression had a significantly shorter DFS than those in any other subgroup $(P=0.007)$. Our study is the first to confirm that SPD-L1 concentration is significantly associated with patient outcome in PTC. Soluble PD-L1 may provide clinicians with a non-invasive biomarker that can lessen dependence on tissue biopsies and diagnose aggressive thyroid cancers at a more treatable stage.
\end{abstract}

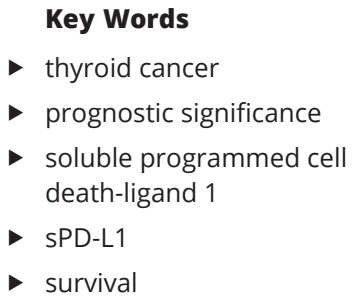

Endocrine Connections (2019) 8, 1040-1051

\section{Introduction}

Thyroid gland carcinoma is the most common endocrine malignancy, irrespective of ethnicity or geographic location, accounting for approximately $90 \%$ of endocrine cancers and $2.1 \%$ of all cancer diagnoses worldwide $(1,2)$. Differentiated thyroid cancers (DTC; both papillary (PTC) and follicular (FTC) histologies), derived from thyroid follicular cells, are the most common subtype, accounting for approximately $90 \%$ of all newly diagnosed cases (2). Standard of care for these patients typically involves surgical resection and suppression of thyroid-stimulating hormone (TSH) via levothyroxine, with additional consideration for adjuvant radioactive iodine (RAI)

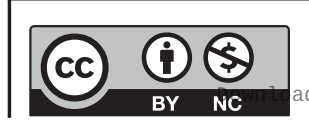

This work is licensed under a Creative Commons Attribution-NonCommercial 4.0 International License. ded from Bioscientifica.com at 04/26/2023 12:15:41PM 
treatment to ablate residual thyroid tissue and eradicate any suspected micrometastases or recurrent disease (1). However, despite initial treatment, approximately 30\% of patients experience a clinically significant recurrence throughout their lifetime (3). Moreover, $5-15 \%$ of patients with metastatic disease will become refractory to RAI, which is associated with a 10-year survival rate of only $10 \%(4,5)$. As mortality rates remain high for patients with advanced or metastatic DTC, continued research exploring novel molecular-based biomarkers that can enhance the capabilities of standard clinicopathological staging, influence treatment direction and optimise patient benefit from novel anti-tumour agents is vital.

Programmed cell death 1 (PD-1) is an immune checkpoint receptor inducibly expressed on activated T, B and natural killer (NK) cells (6). PD-1 interacts with two ligands, programmed cell death-ligand 1 (PD-L1/B7H1/CD274) and programmed cell death-ligand 2 (PD-L2/B7DC/CD273) (7). PD-L1 is expressed on specific tumour and immune cells, including activated $\mathrm{B}$ and $\mathrm{T}$ cells, dendritic cells (DCs) and macrophages, whilst PD-L2 expression is limited to macrophages and DCs. PD-1/PD-L1 interactions impede $\mathrm{T}$ cell proliferation, survival and effector functions and promote the differentiation of $\mathrm{CD} 4+\mathrm{T}$ cells into regulatory $\mathrm{T}$ cells (Tregs), ultimately protecting the tumour from immunemediated rejection. We have recently completed a metaanalysis confirming that elevated tumour PD-L1 is a prognostic indicator for reduced DFS in patients with non-medullary thyroid carcinoma (8).

Checkpoint inhibitors targeting the PD-1/PD-L1 signalling pathway have produced impressive responses in select patients across several tumour types, including advanced melanoma, non-small-cell lung carcinoma and renal cell cancer (RCC) (9). Despite these encouraging results, only a fraction of patients currently benefit from checkpoint blockade therapy (10). Primary resistance to anti-PD-1/PD-L1 therapies has been reported to affect up to $60 \%$ of patients (11). Moreover, such treatments are expensive and broad prescription in the absence of predictive biomarkers is unlikely to be financially sustainable $(12,13)$. Identifying responders and nonresponders prior to the commencement of treatment would considerably reduce patient exposure to avoidable toxicities, and the financial burden of these therapies on healthcare systems.

Elevated sPD-L1 has recently been identified as a poor prognostic factor in several cancer types, including multiple myeloma, diffuse large B-cell lymphoma (DLBCL), RCC, hepatocellular, lung and gastric cancer
$(14,15,16,17,18,19)$. However, to date, the prognostic value of SPD-L1 in thyroid cancer remains to be investigated. We therefore aimed to investigate the prognostic value of soluble PD-L1 in patients with PTC and to evaluate the association between serum and plasma sPD-L1 levels with tumoural PD-L1 expression.

\section{Materials and methods}

\section{Patient characteristics}

Ethics approval was obtained from the South West Sydney Local Health District Human Research Ethics Committee via the Centre for Oncology Education and Research Translation (CONCERT) Biobank, Australia/TCRC/3/0203-2015 (HREC/13/LPOOL/449, HREC/14/LPOOL/152 and HREC/13/LPOOL/158) $(20,21)$.

Between December 2012 and March 2017, serum and plasma samples of 101 patients with confirmed PTC presenting at Liverpool Hospital were included into our study. All patients provided their written informed consent prior to study inclusion. Clinical characteristics were obtained retrospectively. All patients were followed up for survival status until March 2018.

The following clinical factors of PTC patients were examined: age, sex, tumour stage (AJCC/UICC classification 7th edition), tumour size, capsular invasion, extrathyroidal extension, lymphovascular invasion, multicentricity, presence of concurrent lymphocytic thyroiditis and lymph node metastasis.

DFS was defined as the period between completion of primary treatment and detection of residual disease, recurrent disease or death. An excellent response (absence of persistent tumour) following initial therapy was defined as (1) no clinically detectable thyroid cancer, (2) no imaging evidence of tumour by RAI imaging and/or neck ultrasound (US) or (3) low serum thyroglobulin (Tg) during TSH suppression ( $\mathrm{Tg}<0.2 \mathrm{ng} / \mathrm{mL}$ ) or following stimulation $(\mathrm{Tg}<1 \mathrm{ng} / \mathrm{mL})$ with negative thyroglobulin antibodies. The patients that did not experience tumour persistence were censored at the last follow-up contact.

\section{Blood sampling}

Patient blood samples were collected from each subject by CONCERT Biobank staff prior to surgery in vacutainer tubes containing EDTA for plasma or non-additive vacutainers for serum specimens. Specimens were centrifuged at $600 \mathrm{~g}$ for $20 \mathrm{~min}$ at room temperature to separate plasma from whole blood. Serum tubes were centrifuged at $3000 \boldsymbol{g}$ for

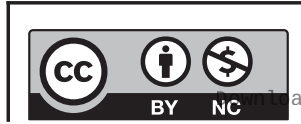

This work is licensed under a Creative Commons Attribution-NonCommercial 4.0 International License. ded from Bioscientifica.com at 04/26/2023 12:15:41PM 
$10 \mathrm{~min}$ at $4^{\circ} \mathrm{C}$. Serum and plasma samples were stored as $500 \mu \mathrm{L}$ aliquots at $-80^{\circ} \mathrm{C}$ until analysis. Peripheral blood samples obtained from healthy controls were collected in the same manner. Healthy controls were defined as volunteers with no active medical conditions.

\section{sPD-L1 ELISAs}

Soluble PD-L1 (sPD-L1) was quantified using a commercially available ELISA (PDCD1LG1 ELISA kit, USCN Life Science, Wuhan, China) according to the manufacturers protocol. The minimum detectable concentration of SPD-L1 was $0.052 \mathrm{ng} / \mathrm{mL}$ in serum and $0.014 \mathrm{ng} / \mathrm{mL}$ in plasma, and the detection range was $0.156-10 \mathrm{ng} / \mathrm{mL}$. Each sample was analysed in duplicate. The intra- and interassay coefficients of variation were below $20 \%$.

\section{Immunohistochemistry}

Small core biopsies (approximately $0.6 \mathrm{~mm}$ in diameter) from formalin-fixed paraffin-embedded (FFPE) tissues with a confirmed diagnosis of PTC were used to construct tissue microarrays. Sections were stained with anti-PD-L1 (clone SP263) rabbit monoclonal primary antibody (Ventana Medical Systems, Tucson, AZ, USA) on the Ventana BenchMark Ultra automated staining platform using the OptiView Detection Kit. Specimens were given a score of 3 if $\geq 50 \%$ tumour cells are PD-L1 positive; 2 if $\geq 5 \%$ but $<50 \%$ tumour cells are PD-L1 positive; 1 if $\geq 1 \%$ but $<5 \%$ of tumour cells are PD-L1 positive and 0 if $<1 \%$ tumour cells express PD-L1. PD-L1 status was considered positive when $\geq 1 \%$ of tumour cells demonstrated membranous staining (score 1, 2 or 3). Each slide was reviewed by two investigators who were blinded to clinical outcome.

\section{Statistical analyses}

Correlations were analysed using the Pearson's chisquared test, Fisher's exact test, Mann-Whitney test or Spearman's rank correlation test. Regarding DFS, receiver-operating characteristic (ROC) curve analysis was performed to determine the optimal cut-off value for sPD-L1 concentration. Survival curves were plotted using the Kaplan-Meier method and compared using the logrank test. A Cox regression model was used to perform multivariate analyses that included all clinicopathological features as covariates. A two-sided $P<0.05$ was considered statistically significant. All statistical analyses were performed using GraphPad Prism v.7.0d and SPSS software (version 22).

\section{Results}

\section{Patient characteristics}

Table 1 summarises the characteristics of the cohort. The median age was 47 years, and majority of cases were female. Twelve patients were treated with hemithyroidectomy and the remaining 89 patients with total thyroidectomy.

Table 1 Summary of patient characteristics.

\begin{tabular}{|c|c|c|}
\hline \multirow[b]{2}{*}{ Patient characteristics } & \multicolumn{2}{|c|}{ Number of patients } \\
\hline & \multicolumn{2}{|c|}{$(n=101)$} \\
\hline \multicolumn{3}{|l|}{ Age } \\
\hline Median (range) & $47.0(20-80)$ & \\
\hline$<55$ & 72 & $71.30 \%$ \\
\hline$\geq 55$ & 29 & $28.70 \%$ \\
\hline \multicolumn{3}{|l|}{$\operatorname{Sex}$} \\
\hline Male & 29 & $28.70 \%$ \\
\hline Female & 72 & $71.30 \%$ \\
\hline \multicolumn{3}{|l|}{ TNM stage } \\
\hline 1 & 74 & $73.30 \%$ \\
\hline II & 5 & $5.00 \%$ \\
\hline III & 16 & $15.80 \%$ \\
\hline IVa & 6 & $5.90 \%$ \\
\hline $\mathrm{IVb}$ & 0 & $0.00 \%$ \\
\hline \multicolumn{3}{|l|}{ Tumour size } \\
\hline Median (range) & $1.1(0.05-7.0)$ & \\
\hline$<2 \mathrm{~cm}$ & 77 & $76.20 \%$ \\
\hline$\geq 2 \mathrm{~cm}$ & 24 & $23.80 \%$ \\
\hline \multicolumn{3}{|l|}{ Multifocality } \\
\hline Present & 36 & $35.60 \%$ \\
\hline Absent & 65 & $64.40 \%$ \\
\hline \multicolumn{3}{|l|}{ Extrathyroidal extension } \\
\hline Present & 23 & $22.80 \%$ \\
\hline Absent & 78 & $77.20 \%$ \\
\hline \multicolumn{3}{|l|}{ Lymphovascular invasion } \\
\hline Present & 24 & $23.80 \%$ \\
\hline Absent & 77 & $76.20 \%$ \\
\hline \multicolumn{3}{|l|}{ Capsular invasion } \\
\hline Present & 13 & $12.90 \%$ \\
\hline Absent & 88 & $87.10 \%$ \\
\hline \multicolumn{3}{|l|}{ Lymph node metastases } \\
\hline Present & 48 & $47.50 \%$ \\
\hline Absent & 53 & $52.50 \%$ \\
\hline \multicolumn{3}{|c|}{ Concurrent Hashimoto's thyroiditis } \\
\hline Present & 35 & $34.70 \%$ \\
\hline Absent & 66 & $65.30 \%$ \\
\hline \multicolumn{3}{|l|}{ PTC subtype } \\
\hline СРTC & 76 & $75.20 \%$ \\
\hline FVPTC & 12 & $11.90 \%$ \\
\hline PTC/FTC & 7 & $6.90 \%$ \\
\hline Diffuse sclerosing variant & 2 & $2.00 \%$ \\
\hline Oncocytic variant & 2 & $2.00 \%$ \\
\hline Solid variant & 1 & $1.00 \%$ \\
\hline $\mathrm{PTC} / \mathrm{MTC}$ & 1 & $1.00 \%$ \\
\hline \multicolumn{3}{|l|}{ Treatment } \\
\hline Hemithyroidectomy & 12 & $11.90 \%$ \\
\hline Total thyroidectomy & 89 & $88.10 \%$ \\
\hline
\end{tabular}

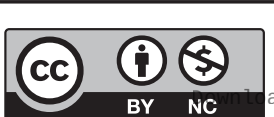


Histologically, the majority of patients (75.2\%) were diagnosed with classic form PTC (CPTC).

\section{Serum sPD-L1 level is increased in PTC patients}

The median serum sPD-L1 concentration in the healthy cohort and PTC patients was $0.37 \mathrm{ng} / \mathrm{mL}$ (range $0.30-0.79$, mean $0.44 \pm 0.17$ ) and $0.48 \mathrm{ng} / \mathrm{mL}$ (range 0.05-4.91, mean $0.65 \pm 0.77$ ), respectively. Soluble levels of PD-L1 in the serum of PTC patients were significantly higher when compared with levels identified in healthy individuals $(P=0.028$, Fig. 1$)$. Ten $(9.9 \%)$ of the PTC patients had serum sPD-L1 levels below the detection range $(0.156-10 \mathrm{ng} / \mathrm{mL})$.

\section{Plasma SPD-L1 level is lower than serum SPD-L1 in PTC patients}

The median plasma sPD-L1 concentration in PTC patients was $0.21 \mathrm{ng} / \mathrm{mL}$ (range $0.01-1.71$, mean $0.30 \pm 0.25$ ), whilst the median plasma sPD-L1 levels in the healthy cohort

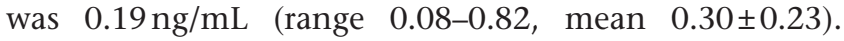
No significant difference between plasma sPD-L1 levels in PTC patients and healthy individuals was observed ( $P=0.9273$, Fig. 1). The median level of serum sPD-L1 was significantly higher than plasma sPD-L1 levels $(0.48 \mathrm{ng} / \mathrm{mL}$ vs $0.21 \mathrm{ng} / \mathrm{mL}, \quad P<0.0001)$. No significant correlation was observed between serum and plasma sPD-L1 levels (Supplementary Fig. 1, see section on supplementary data given at the end of this article; $r=0.136, P=0.176$ ).

\section{Cut-off value for SPD-L1}

There is currently no defined cut-off value to distinguish between high and low sPD-L1 levels in patients with cancer. Prior studies exploring the prognostic capabilities of sPD-L1 concentration have used the median sPD-L1 level in the analysed cancer patient cohort (22), median sPD-L1 level in a corresponding healthy cohort or ROC curve models $(23,24)$ to establish a cut-off value. We therefore used both the median serum and plasma sPD-L1 value in the healthy cohort $(0.37 \mathrm{ng} / \mathrm{mL}$ and $0.19 \mathrm{ng} / \mathrm{mL}$, respectively), and the median serum and plasma sPD-L1 value in the PTC patient cohort $(0.48 \mathrm{ng} / \mathrm{mL}$ and $0.21 \mathrm{ng} / \mathrm{mL}$, respectively) as the cut-off value to determine if patients had high or low sPD-L1 levels. Additionally, ROC curves were generated to determine the optimal sPD-L1 cut-off value for determining DFS (Supplementary Fig. 2).

\section{Serum SPD-L1 level correlated with clinicopathological features of PTC}

The associations between the serum and plasma levels of SPD-L1 and the clinicopathological features of the
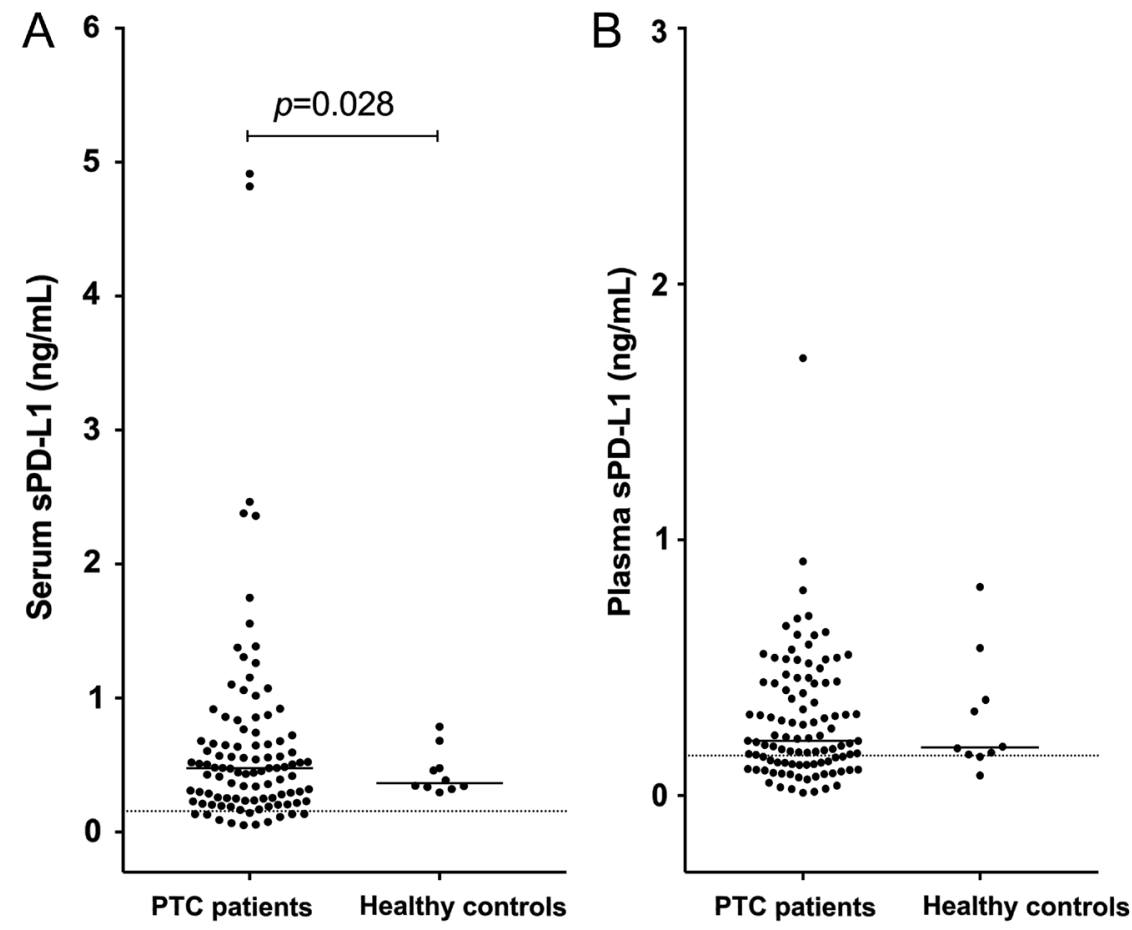

\section{Figure 1}

Serum (A) and plasma (B) SPD-L1 levels in PTC patients and healthy individuals. The dashed line represents the detection limit of the ELISA $(0.156 \mathrm{ng} / \mathrm{mL})$. The horizontal lines signify the medians. https://ec.bioscientifica.com https://doi.org/10.1530/EC-19-0210 (c) 2019 The authors Published by Bioscientifica Ltd
This work is licensed under a Creative Commons Attribution-NonCommercial 4.0 International License. ded from Bioscientifica com at 04/26/2023 12:15:41PM 
patients are summarised in Table 2 and Supplementary Table 1. No correlations were noted between sPD-L1 level and age, TNM stage, multifocality, lymphovascular invasion, capsular invasion, lymph node metastasis or concurrent Hashimoto's thyroiditis. However, presence of extrathyroidal extension was significantly associated with an increase in serum sPD-L1 level (Table 2; $P=0.015$ ). Moreover, male patients were more likely to present with elevated serum sPD-L1 levels (Supplementary Table 1; $P=0.047)$.

\section{DFS curves using median SPD-L1 as the cut-off value}

At a median follow-up time of 16 months (range 1-60 months), disease progression occurred in 20 patients at a median of 13 months (range 2-39 months). On univariate analysis, patients with high serum sPD-L1 levels demonstrated a significantly shorter DFS compared with those with low sPD-L1 levels; this was significant when using both the median cut-off value from the healthy cohort (Fig. 2; 14 months vs 21.5 months, $P=0.014$ ) as well as the median cut-off value from the patient cohort (Fig. 2; 14 months vs 22 months, $P=0.011$ ). However, plasma sPD-L1 was not a significant predictive marker for DFS using either cut-off value (Fig. $2 ; P=0.263, P=0.405$ ).

\section{Optimal cut-off for SPD-L1 derived from ROC curve}

The optimal cut-off for serum sPD-L1 derived from the ROC curve was $0.44 \mathrm{ng} / \mathrm{mL}$, with an area under the curve of 0.647 (95\% confidence interval: $0.521-0.773, P=0.043$,

Table 2 Relationship of serum and plasma SPD-L1 expression to clinicopathological characteristics in 101 PTC cases.

\begin{tabular}{|c|c|c|c|c|c|c|}
\hline \multirow[b]{2}{*}{ Patient characteristics } & \multicolumn{3}{|c|}{ Serum SPD-L1 expression score Healthy cohort cut-off } & \multicolumn{3}{|c|}{ Plasma SPD-L1 expression Healthy cohort cut-off } \\
\hline & SPD-L1 low $(<0.37 \mathrm{ng} / \mathrm{mL})$ & $\mathrm{sPD}-\mathrm{L} 1^{\text {high }}(\geq 0.37 \mathrm{ng} / \mathrm{mL})$ & $P$ value & SPD-L1 low $(<0.19 \mathrm{ng} / \mathrm{mL})$ & SPD-L1 high $(\geq 0.19 \mathrm{ng} / \mathrm{mL})$ & $P$ value \\
\hline \multicolumn{7}{|l|}{ Age } \\
\hline$<55$ & 29 & 43 & & 30 & 42 & \\
\hline$\geq 55$ & 12 & 17 & 1 & 10 & 19 & 0.653 \\
\hline \multicolumn{7}{|l|}{ Sex } \\
\hline Male & 8 & 21 & & 8 & 21 & \\
\hline Female & 33 & 39 & 0.118 & 32 & 40 & 0.177 \\
\hline \multicolumn{7}{|l|}{ TNM stage } \\
\hline$|/| \mid$ & 33 & 46 & & 31 & 48 & \\
\hline II/IV & 8 & 14 & 0.807 & 9 & 13 & 1 \\
\hline \multicolumn{7}{|l|}{ Tumour size } \\
\hline$<2 \mathrm{~cm}$ & 32 & 45 & & 33 & 44 & \\
\hline$\geq 2 \mathrm{~cm}$ & 9 & 15 & 0.814 & 7 & 17 & 0.339 \\
\hline \multicolumn{7}{|l|}{ Multifocality } \\
\hline Present & 15 & 20 & & 18 & 17 & \\
\hline Absent & 26 & 40 & 0.832 & 22 & 44 & 0.09 \\
\hline \multicolumn{7}{|l|}{ Extrathyroidal extension } \\
\hline Present & 4 & 19 & & 7 & 16 & \\
\hline Absent & 37 & 41 & 0.015 & 33 & 45 & 0.342 \\
\hline \multicolumn{7}{|l|}{ Lymphovascular invasion } \\
\hline Present & 7 & 17 & & 12 & 12 & \\
\hline Absent & 34 & 43 & 0.238 & 28 & 49 & 0.244 \\
\hline \multicolumn{7}{|l|}{ Capsular invasion } \\
\hline Present & 8 & 5 & & 3 & 10 & \\
\hline Absent & 33 & 55 & 0.132 & 37 & 51 & 0.236 \\
\hline \multicolumn{7}{|l|}{ Lymph node metastases } \\
\hline Present & 19 & 29 & & 18 & 30 & \\
\hline Absent & 22 & 31 & 1 & 22 & 31 & 0.69 \\
\hline \multicolumn{7}{|c|}{ Concurrent Hashimoto's thyroiditis } \\
\hline Present & 12 & 23 & & 12 & 23 & \\
\hline Absent & 29 & 36 & 0.395 & 28 & 37 & 0.521 \\
\hline \multicolumn{7}{|l|}{ Treatment } \\
\hline Total thyroidectomy & 38 & 51 & & 35 & 54 & \\
\hline Hemithyroidectomy & 3 & 9 & 0.351 & 5 & 7 & 1 \\
\hline
\end{tabular}

The median SPD-L1 concentration in the serum $(0.37 \mathrm{ng} / \mathrm{mL})$ and plasma $(0.19 \mathrm{ng} / \mathrm{mL})$ of the healthy cohort was used as the cut-off value. Bold indicates statistical significance.

https://ec.bioscientifica.com

https://doi.org/10.1530/EC-19-0210 (c) 2019 The authors Published by Bioscientifica Ltd

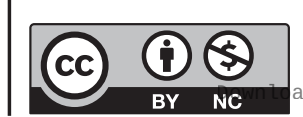

This work is licensed under a Creative Commons Attribution-NonCommercial 4.0 International License. ded from Bioscientifica.com at $04 / 26 / 2023$ 12:15:41PM via free access 
A

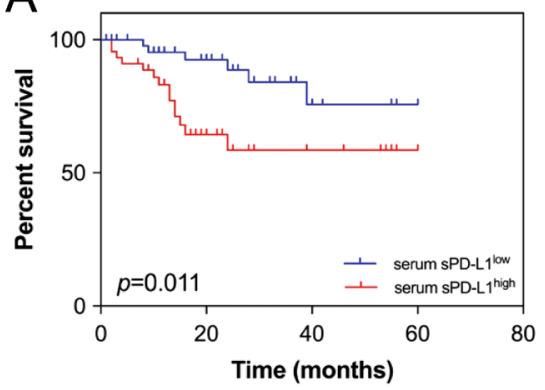

C

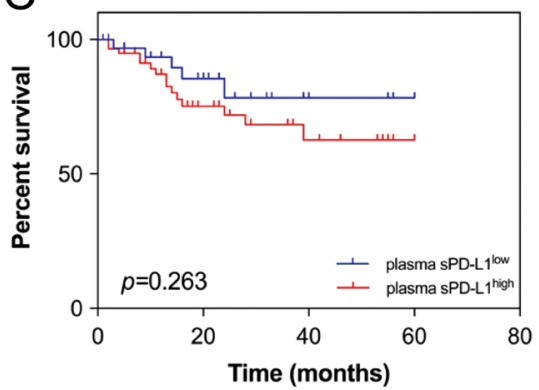

B

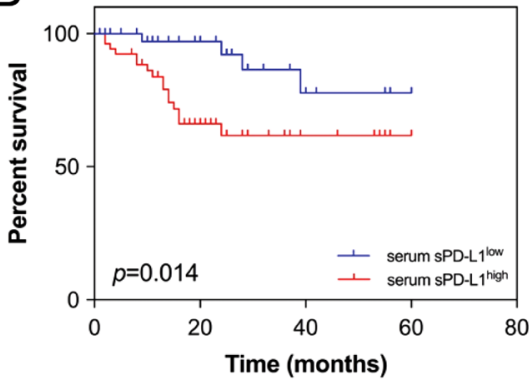

D

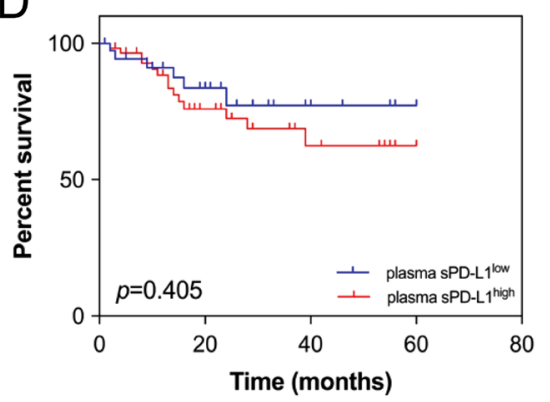

\section{Figure 2}

(A) Kaplan-Meier DFS analysis using the cut-off point for serum SPD-L1 at $0.48 \mathrm{ng} / \mathrm{mL}$. (B) Kaplan-Meier DFS analysis using the cut-off point for serum SPD-L1 at $0.37 \mathrm{ng} / \mathrm{mL}$. (C) Kaplan-Meier DFS analysis using the cut-off point for plasma sPD-L1 at $0.19 \mathrm{ng} / \mathrm{mL}$. (D) Kaplan-Meier DFS analysis using the cut-off point for plasma SPD-L1 at $0.21 \mathrm{ng} / \mathrm{mL}$.
Supplementary Fig. 2A). This provided positive and negative predictive values of 75 and $48 \%$, respectively. For plasma sPD-L1, the optimal cut-off was $0.19 \mathrm{ng} / \mathrm{mL}$, with an area under the curve of 0.564 (95\% confidence interval: $0.435-0.694, P=0.375$, Supplementary Fig. 2B). This provided positive and negative predictive values of 75 and 60\%, respectively. Survival analysis revealed that the patients with high serum sPD-L1 had significantly shorter DFS compared with those with low sPD-L1 levels (14 months vs 23 months, $P=0.010$, Table 3 ). PTC patients with high plasma sPD-L1 also showed shorter DFS than those with low plasma sPD-L1, but the difference was not significant (15 months vs 19 months, $P=0.263$ ).

\section{SPD-L1 is an independent risk factor for DFS in PTC patients}

To further analyse sPD-L1 level as prognostic parameter, a multivariate Cox regression model was performed. Variables including age ( $<55$ years vs $\geq 55$ years), gender (male vs female), TNM stage (I/II vs III/IV), tumour size ( $<2 \mathrm{~cm}$ vs $\geq 2 \mathrm{~cm}$ ), multifocality (present vs absent), extrathyroidal extension (present vs absent), lymphovascular invasion (present vs absent), capsular invasion (present vs absent), lymph node metastasis (present vs absent), concurrent Hashimoto's thyroiditis (present vs absent) and treatment type (total thyroidectomy vs hemithyroidectomy) were included in the model.

When using the cut-off values derived from the ROC curves, multivariate analysis confirmed that elevated serum sPD-L1 levels were significantly associated with shorter DFS ( $P=0.017$, Table 3$)$. Increased serum sPD-L1 was also significantly associated with a shorter DFS when the cut-off values from the healthy and patient cohort were implemented (data not shown). Serum sPD-L1 was the only remaining patient characteristic predictive of DFS on multivariate analysis (Table 3 ).

\section{Correlation between serum and plasma sPD-L1 levels with tumoural PD-L1 expression}

Sixty-five of the 101 PTC patients enrolled in our study had FFPE tissue available for analysis. Of the 65 patients, 30 were considered positive for membranous PD-L1 expression. Representative examples of PD-L1 scoring are shown in Fig. 3. No significant association was found between serum $(P=0.652)$ or plasma $(P=0.771)$ PD-L1 levels and tumoural PD-L1 expression. The 65 patients included were representative of the total cohort of 101 cases, with significantly decreased DFS observed in patients with positive serum sPD-L1 concentration $(P=0.039)$. Tumoural PD-L1 was not a significant predictive biomarker of DFS in our cohort (11.5 months vs 16 months, $P=0.256$ ).

Sub-analysis was performed to assess the relationship between tumoural PD-L1 expression, soluble PD-L1 levels and prognosis. Cases were divided into four groups based on positive or negative tumoural PD-L1 expression and high or low serum sPD-L1 (group 1: tumoural PD-L1 ${ }^{\text {low }}$, serum sPD-L1 ${ }^{\text {low }}$ (15 patients); group 2: tumoural
This work is licensed under a Creative Commons Attribution-NonCommercial 4.0 International License. ded from Bioscientifica.com at 04/26/2023 12:15:41PM via free access 
Table 3 Univariate and multivariate analyses of parameters associated with DFS using the ROC cut-off.

\begin{tabular}{l}
\hline Patient characteristics \\
\hline Age \\
$<55$ vs $\geq 55$ \\
Sex \\
Male vs female \\
TNM stage \\
I/II vs II/IV \\
Tumour size \\
$<2$ cm vs $\geq 2$ cm \\
Multifocality \\
Present vs absent \\
Extrathyroidal extension \\
Present vs absent \\
Lymphovascular invasion \\
Present vs. absent \\
Capsular invasion \\
Present vs absent \\
Lymph node metastases \\
Present vs absent \\
Concurrent Hashimoto's thyroiditis \\
Present vs absent \\
Treatment \\
Total thyroidectomy vs hemithyroidectomy \\
Serum sPD-L1 low ( $<0.44$ ng/mL) ROC \\
Low vs high \\
Plasma sPD-L1 1 low ( $<0.19$ ng/mL) ROC \\
Low vs high \\
\hline
\end{tabular}

\begin{tabular}{c}
\hline Univariate analysis \\
\hline$P$ value \\
\hline 0.46 \\
$\mathbf{0 . 0 3 8}$ \\
0.353 \\
0.471 \\
0.571 \\
0.165 \\
0.074 \\
0.858 \\
0.244 \\
0.484 \\
0.374 \\
$\mathbf{0 . 0 1 0}$ \\
0.263
\end{tabular}

\begin{tabular}{|c|c|c|}
\hline \multicolumn{3}{|c|}{ Multivariate analysis } \\
\hline $\mathrm{HR}$ & $95 \% \mathrm{Cl}$ & $P$ value \\
\hline 0.401 & $0.103-1.563$ & 0.188 \\
\hline \multirow[t]{2}{*}{1.389} & $0.468-4.122$ & 0.554 \\
\hline & $0.534-5.403$ & 0.37 \\
\hline 0.798 & $0.254-2.503$ & 0.698 \\
\hline 0.937 & $0.299-2.939$ & 0.911 \\
\hline 1.516 & $0.482-4.772$ & 0.477 \\
\hline 3.241 & $0.986-10.651$ & 0.053 \\
\hline 3.402 & $0.774-14.947$ & 0.105 \\
\hline 1.531 & $0.435-5.383$ & 0.507 \\
\hline 0.46 & $0.154-1.372$ & 0.163 \\
\hline 3.921 & $0.785-19.581$ & 0.096 \\
\hline 4.196 & $1.296-13.590$ & 0.017 \\
\hline 1.946 & $0.599-6.319$ & 0.268 \\
\hline
\end{tabular}

Bold indicates statistical significance.

PD-L1 ${ }^{\text {high }}$, serum sPD-L1 ${ }^{\text {low }}$ (14 patients); group 3: tumoural PD-L1 ${ }^{\text {low }}$, serum sPD-L1 ${ }^{\text {high }}$ (17 patients); group 4: tumoural PD-L1 $1^{\text {high }}$, serum sPD-L1 ${ }^{\text {high }}$ (19 patients)). The median serum sPD-L1 value from the PTC patient cohort $(0.48 \mathrm{ng} / \mathrm{mL})$ was used as the cut-off on the basis that it remained the most significant predictive marker on multivariate analysis. Patients with both positive serum and tumoural PD-L1 expression had a significantly shorter DFS than those in any other subgroup (Fig. $3 ; P=0.007$ ).

\section{Discussion}

Molecular markers may assist clinicians in more accurately discriminating between low-, intermediate- and high-risk DTC (3). Accurate risk prediction would inform therapeutic decision making, such as surgical extent or treatment with novel PD-1/PD-L1-targeted immunotherapies. Given the limited predictive value of PD-L1 expression by IHC, additional biomarkers are currently being explored (25). Accumulating evidence suggests that levels of sPD-L1 in the serum, plasma or both, of cancer patients may predict certain clinicopathological characteristics, response to treatment and survival outcomes $(14,15,17,18,19,22$, $24,26,27,28,29)$. There are a number of reasons why sPD-L1 may provide different information and potentially a broader measure of systemic inflammation state than tumour PD-L1 levels and these are discussed further below.

In the present study, we investigated both serum and plasma levels of sPD-L1 in a series of 101 PTC patients to identify any associations with clinicopathological characteristics and DFS. Serum sPD-L1 concentrations in PTC patients were significantly higher when compared to healthy controls $(P=0.029)$. Elevated levels of sPD-L1 have similarly been reported in patients with advanced NSCLC, melanoma and gastric cancer (14, 19, 29). However, lower median levels of sPD-L1 were reported in patients with hepatocellular carcinoma (HCC) compared to healthy controls $(0.5 \mathrm{ng} / \mathrm{mL}$ vs $0.78 \mathrm{ng} / \mathrm{mL} ; P<0.01)$ (17). Recently, Boffa et al. characterised PD-L1 expression in the peripheral blood of lung cancer patients using single-cell, automated slide-based digital pathology (30). Two populations were included as controls; (1) a cohort of 'healthy controls' including volunteers with no active medical conditions and (2) a second cohort of 'unhealthy controls' comprising patients initially suspected of having 

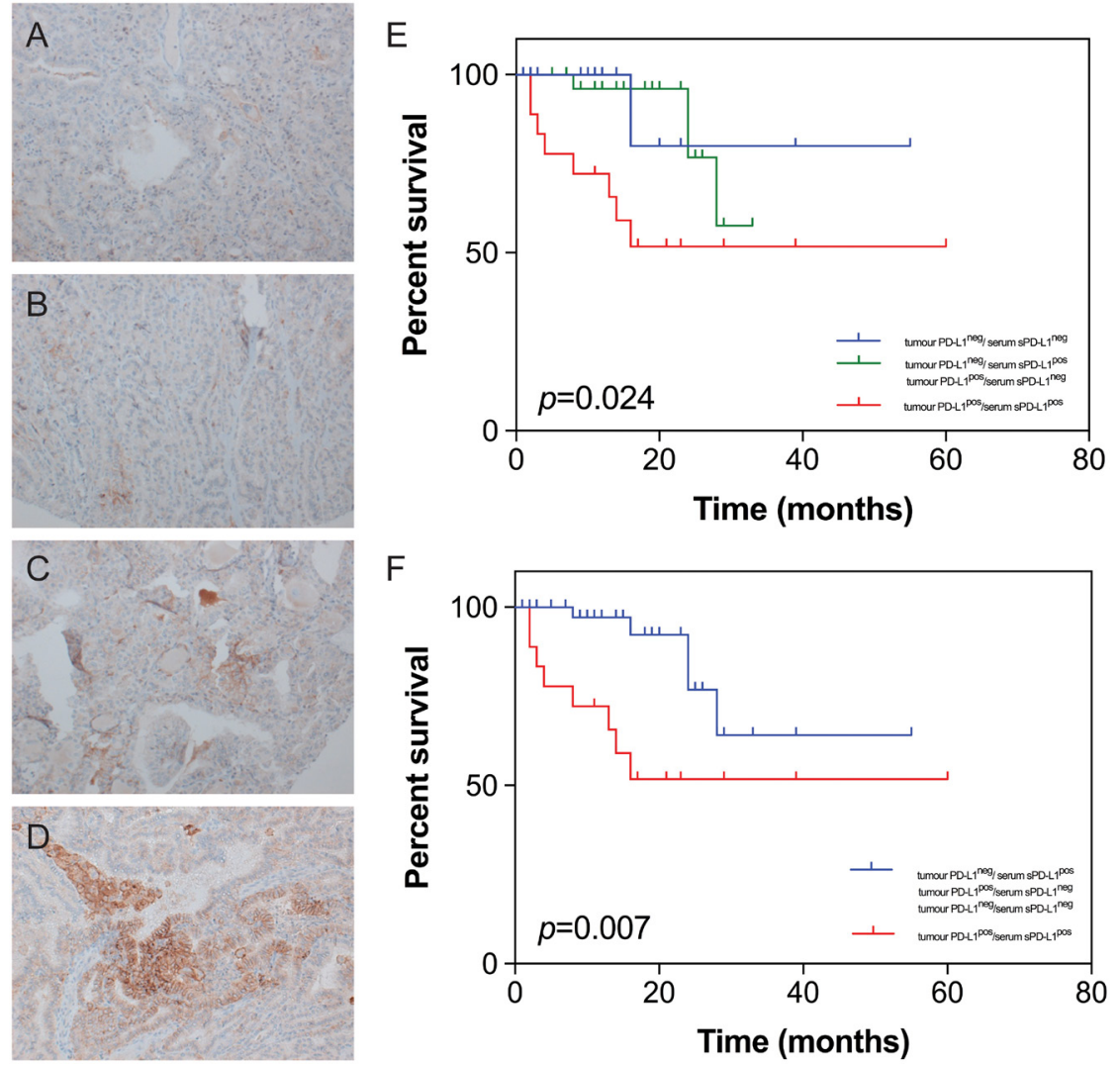
Figure 3
Immunohistochemical (IHC) staining pattern for tumoural PD-L1 expression. Representative examples of score $0(A)$, score $1(B)$, score 2 (C) and score 3 (D). Kaplan-Meier DFS analysis of patients with high or low tumoural and serum PD-L1 expression using three subgroups (E) and two subgroups (F).

lung cancer, but were ultimately deemed not to have lung cancer. Whilst no PD-L1 expression was detected within peripheral circulating cells associated with malignancy (CCAMs) in healthy controls, they were detected in $20 \%$ of the 'unhealthy controls', suggesting that PD-L1 expression may be influenced by a number of additional factors unrelated to cancer.

In PTC patients, elevated serum sPD-L1 levels were significantly associated with an increased incidence of extrathyroidal extension. Furthermore, PTC patients with higher serum sPD-L1 concentration had a poorer DFS than those with low sPD-L1 levels $(P=0.011)$. Multivariate analysis confirmed that a higher sPD-L1 level was a significant independent prognostic factor for reduced DFS $(P=0.020)$. We went on to establish that the combination of PD-L1 concentration in both serum and tissue was a more potent predictor of DFS than either marker alone $(P=0.007)$. Several studies have similarly observed a significant association between elevated sPD-L1 levels and disease progression in other tumour types, including NSCLC, HCC, biliary tract carcinoma, multiple myeloma and DLBCL $(14,15,18,23,26)$. Conversely, no significant difference in sPD-L1 concentration was detected in the serum of patients with cervical cancer, patients with cervical intraepithelial neoplasia (CIN) and healthy controls (31). Moreover, higher levels of sPD-L1 were associated with differentiated cancer, an absence of lymph node metastasis and improved overall survival in a cohort of advanced gastric cancer patients (32). Levels of sPD-1 and SPD-L1 were not indicative of an adverse outcome in a cohort of 41 patients with advanced pancreatic cancer (pancreatic ductal adenocarcinoma (PDACs): $n=40$, acinar cell carcinoma $n=1$ ) (22). Membranous PD-L1 has been reported to be poorly expressed in PDACs; thus, the low concentration of sPD-L1 in the serum of PDAC patients may reflect its minimal expression within the tumour microenvironment.

The exact origin of sPD-L1 in patients with cancer remains unclear. It has been postulated that circulating sPD-L1 may arise from intrinsic splicing activities in tumour cells and the proteolytic cleavage of membranebound PD-L1. Whilst SPD-L1 was detected in the supernatants of membrane PD-L1+ myeloma cell lines, it was not observed in the PD-L1-negative cell lines, suggesting that cell-surface PD-L1 may be a source of sPD-L1 (14). The correlation between serum sPD-L1 levels and PD-L1 expression in the tumour tissue of patients with nasal natural killer/T cell lymphoma (NNKTL) was also reported to be statistically significant (24). However, the tumour may not be the only source of sPD-L1.

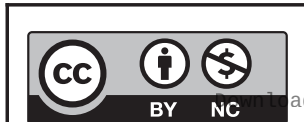


Rossille et al. reported no association between plasma sPD-L1 and tumoural PD-L1 expression in DLBCL patients (15). Similarly, no correlation was found between levels of serum SPD-L1 and tumoural PD-L1 expression in a cohort of patients with advanced pancreatic cancer (22). We too established no significant relationship between tumoural PD-L1 expression and soluble PD-L1 levels in either serum or plasma. Cumulatively, these results suggest that nonmalignant cells may produce sPD-L1. Soluble PD-L1 was shown to be secreted from human myeloid dendritic cells (DCs) in the presence of cytokines and LPS during DC maturation $(29,33)$. Moreover, myeloid derived cells have the capacity to release PD-L1 molecules, whilst T cells, even when optimally stimulated, only contribute trace amounts (33). Therefore, sPD-L1 is derived from a range of sources, both within the tumour and the immune system.

Circulating sPD-L1 retains its ligand-binding immunoglobulin variable domain (IgV) (33). Binding of sPD-L1 to PD-1 located on circulating T cells in peripheral blood may suppress their anti-tumour activity on a systemic scale. The co-incubation of CD4+ and CD8+ T lymphocytes with mature DC-derived SPD-L1 has been shown to promote $\mathrm{T}$ cell apoptosis (33). Moreover, it was recently reported that mesenchymal stromal cells (MSCs) suppress $\mathrm{T}$ cells through the secretion of both PD-1 ligands PD-L1 and PD-L2 (34). Exposure of MSCs to the pro-inflammatory cytokines interferon $\gamma$ (IFN- $\gamma$ ) and tumour necrosis factor $\alpha$ (TNF- $\alpha$ ) upregulated both cell surface and secreted forms of these ligands. Blocking experiments confirmed their role in suppressing $\mathrm{T}$ cell proliferation, interleukin-2 (IL-2) secretion, inducing hypo-responsiveness and cell death. Thus, soluble PD-1 ligands play an important role in modulating $\mathrm{T}$ cell behaviour and induction of peripheral tolerance. Additional research exploring the mechanisms regulating PD-L1 release will be necessary to further optimise PD-L1 checkpoint immunotherapies.

Blockade of the PD-1/PD-L1 axis has produced clinical benefits in patients with a variety of cancers (35). The FDA has approved the anti-PD-1 antibodies nivolumab and pembrolizumab, as well as the PD-L1 inhibitors atezolizumab, avelumab and durvalumab, for the treatment of many diverse cancers, including Merkel cell carcinoma (MCC), classical Hodgkin lymphoma (cHL), urothelilal carcinoma, squamous cell carcinoma of the head and neck (SCCHN), non-small-cell lung carcinoma (NSCLC), RCC and melanoma (36). Potential applications of these immune checkpoint antibodies in thyroid cancer are currently being investigated in a number of ongoing clinical trials. According to results from the KEYNOTE-028 trial presented at the 2016 ASCO Annual Meeting, pembrolizumab has demonstrated promising antitumor activity with durable responses in patients with advanced (unresectable and/or metastatic) PTC and FTC (37). Partial responses at the time of last follow-up were demonstrated in only $9.1 \%(2 / 22)$ of the cohort. However, $12 / 22$ (54.5\%) patients were able to maintain stable disease for prolonged periods with no treatment-associated discontinuations or deaths. In a case study involving a 52-year-old male with ATC, treatment with the BRAF inhibitor vemurafenib achieved a mixed response; however, the incorporation of nivolumab into the treatment regimen resulted in substantial regression of disease (37). The patient continued to remain in complete radiographic and clinical remission 20 months after the initiation of treatment with nivolumab. Melanoma patients with high pre-treatment levels of sPD-L1 had an increased likelihood of progressive disease following treatment by CTLA- 4 or PD-1 blockade (29). The soluble form of PD-1 (sPD-1) also possesses predictive capabilities in NSCLC (27) and hepatitis B virus (HBV)-related hepatocellular carcinoma (HCC) (38) and is currently being investigated in a clinical trial exploring its use as a response-related biomarker in advanced melanoma patients (NCT03197636). Further research will be necessary to elucidate the value of sPD-L1 as a predictive biomarker of PD-1/PD-L1 checkpoint blockade in thyroid cancer. By identifying responders and non-responders prior to the commencement of treatment, patient exposure to avoidable immune-related toxicities and the financial burden of these therapies on healthcare systems would be significantly reduced.

To date, there is a lack of consensus regarding which blood fraction is best for sPD-L1 analysis. Our findings demonstrate that levels of sPD-L1 are not consistent amongst blood fractions collected from patients with PTC, with levels of sPD-L1 in plasma samples significantly lower than those in serum samples. Rapid proteolytic degradation in plasma was observed to occur within minutes of blood sample collection via endogenous proteases and peptidases, resulting in preanalytical variability that may interrupt meaningful data interpretation (39). Mixing of protease inhibitors with blood immediately during the blood draw was recommended to immediately minimise proteolytic degradation. It is important to note that the instruction manual accompanying the ELISA kit chosen for our study highly recommended the use of serum instead of plasma based on their in-house data. However, a number of prior studies have detected elevated sPD-L1 levels in plasma specimens using the same manufacturer's kit $(15,18,28)$. This discrepancy may be due to a lack of

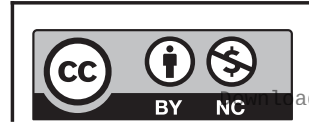

This work is licensed under a Creative Commons Attribution-NonCommercial 4.0 International License. ded from Bioscientifica.com at 04/26/2023 12:15:41PM 
standardisation of protocols regarding sample processing and protein measurement. Processing of plasma at the CONCERT Biobank, from which our samples were obtained, was performed under different conditions to those recommended from the ELISA kit manufacturer. Whilst the CONCERT Biobank protocol specifies for samples to be centrifuged at $600 \mathrm{~g}$ for $20 \mathrm{~min}$ at $20^{\circ} \mathrm{C}$ to separate plasma, the guidelines from the manufacturer state samples be centrifuged for $15 \mathrm{~min}$ at $1000 \mathrm{~g}$ at $2-8^{\circ} \mathrm{C}$. Thus, proteolytic activity may have been greater at a higher temperature, and the centrifugations speed may not have been optimal to isolate the target protein from plasma (40).

The expression of PD-L1 evaluated by IHC is currently the primary biomarker used in determining patient response to immunotherapy treatment (41). However, several concerns have been raised regarding its use. To date, different PD-L1 assays and testing platforms have been incorporated in numerous studies, with each adopting varying scoring algorithms and anti-PD-L1 antibodies (42). Nevertheless, the PD-L1 monoclonal antibodies SP142, E1L3N, 9A11, SP263, 22c3 and 28-8 were recently shown to all yield highly concordant results in 30 cases of NSCLC (43). It was suggested that the discordance seen between studies is instead likely due to the intratumoral and temporal heterogeneity of PD-L1 expression and other assay-specific variables. The incorporation of additional biomarkers, including tumour mutational load, the presence and type of tumour-infiltrating immune cells, additional immune checkpoints and soluble PD-L1 levels may overcome the limitations of PD-L1 testing via IHC $(44,45)$.

Whilst our results suggest that sPD-L1 levels are predictive of DFS in PTC, additional prospective studies with larger sample sizes should be conducted to validate these findings. Additionally, whilst ELISA detected total levels of sPD-L1 in our samples, it was not capable of establishing the quality of sPD-L1, particularly its PD-1binding capacity. Takeuchi et al. recently developed a novel ELISA system for the detection and quantification of SPD-L1 which has retained its PD-1-binding capabilities (bsPD-L1) (46). Whilst the conventional ELISA detected sPD-L1 in only $10.6 \%$ of samples, their new system could determine levels with much higher sensitivity and frequency, with bsPD-L1 identified in 29 of the NSCLC patients (38.6\%). Research clarifying its efficacy and application in other cancer types is warranted.

Our study is the first to confirm that elevated serum sPD-L1 concentration is significantly associated with DFS in PTC. Levels of sPD-L1 may provide clinicians with a non-invasive biomarker that can lessen dependence on tissue biopsies and identify aggressive thyroid cancers at an earlier stage. Serum sPD-L1 may be used to predict favourable clinical response to PD-1/PD-L1 checkpoint inhibitors in PTC.

\section{Supplementary data}

This is linked to the online version of the paper at https://doi.org/10.1530/ EC-19-0210.

\section{Declaration of interest}

The authors declare that there is no conflict of interest that could be perceived as prejudicing the impartiality of the research reported.

\section{Funding}

This research was funded by the Ingham Institute for Applied Medical Research CTC Head \& Neck Research Grant. T L R is the recipient of a Cancer Institute New South Wales Future Research Leader Fellowship. M J A is the recipient of a Western Sydney University Postgraduate Scholarship.

\section{References}

1 Nguyen QT, Lee EJ, Huang MG, Park YI, Khullar A \& Plodkowski RA. Diagnosis and treatment of patients with thyroid cancer. American Health and Drug Benefits 20158 30-40.

2 Kitahara CM \& Sosa JA. The changing incidence of thyroid cancer. Nature Reviews: Endocrinology 201612 646-653. (https://doi. org/10.1038/nrendo.2016.110)

3 Omry-Orbach G. Risk stratification in differentiated thyroid cancer: an ongoing process. Rambam Maimonides Medical Journal 20167 e0003. (https://doi.org/10.5041/RMMJ.10230)

4 Durante C, Haddy N, Baudin E, Leboulleux S, Hartl D, Travagli JP, Caillou B, Ricard M, Lumbroso JD, De Vathaire F, et al. Long-term outcome of 444 patients with distant metastases from papillary and follicular thyroid carcinoma: benefits and limits of radioiodine therapy. Journal of Clinical Endocrinology and Metabolism 200691 2892-2899. (https://doi.org/10.1210/jc.2005-2838)

5 Worden F. Treatment strategies for radioactive iodine-refractory differentiated thyroid cancer. Therapeutic Advances in Medical Oncology 20146 267-279. (https://doi.org/10.1177/1758834014548188)

6 Riella LV, Paterson AM, Sharpe AH \& Chandraker A. Role of the PD-1 pathway in the immune response. American Journal of Transplantation 201212 2575-2587. (https://doi.org/10.1111/j.16006143.2012.04224.x)

7 Francisco LM, Sage PT \& Sharpe AH. The PD-1 pathway in tolerance and autoimmunity. Immunological Reviews 2010236 219-242. (https://doi.org/10.1111/j.1600-065X.2010.00923.x)

8 Aghajani M, Graham S, McCafferty C, Shaheed CA, Roberts T, DeSouza P, Yang T \& Niles N. The clinicopathological and prognostic significance of programmed cell death ligand 1 (PD-L1) expression in patients with non-medullary thyroid cancer: a systematic review and meta-analysis. Thyroid 201828 349-361. (https://doi.org/10.1089/ thy.2017.0441)

9 Topalian SL, Hodi FS, Brahmer JR, Gettinger SN, Smith DC, McDermott DF, Powderly JD, Carvajal RD, Sosman JA, Atkins MB, et al. Safety, activity, and immune correlates of anti-PD-1 antibody in cancer. New England Journal of Medicine 2012366 2443-2454. (https://doi.org/10.1056/NEJMoa1200690)

10 Zhao L, Li C, Liu F, Zhao Y, Liu J, Hua Y, Liu J, Huang J \& Ge C. A blockade of PD-L1 produced antitumor and antimetastatic effects 
in an orthotopic mouse pancreatic cancer model via the PI3K/ Akt/mTOR signaling pathway. OncoTargets and Therapy 201710 2115-2126. (https://doi.org/10.2147/OTT.S130481)

11 O'Donnell JS, Long GV, Scolyer RA, Teng MW \& Smyth MJ. Resistance to PD1/PDL1 checkpoint inhibition. Cancer Treatment Reviews 201752 71-81. (https://doi.org/10.1016/j.ctrv.2016.11.007)

12 Goeree R, Villeneuve J, Goeree J, Penrod JR, Orsini L \& Tahami Monfared AA. Economic evaluation of nivolumab for the treatment of second-line advanced squamous NSCLC in Canada: a comparison of modeling approaches to estimate and extrapolate survival outcomes. Journal of Medical Economics 201619 630-644. (https:// doi.org/10.3111/13696998.2016.1151432)

13 National Institute for Health and Care Excellence. Nivolumab for previously treated locally advanced or metastatic squamous nonsmall-cell lung cancer April 17, 2018. London, UK: NICE, 2018. (available at: https://s3.amazonaws.com/assets.fiercemarkets.net/ public/005-LifeSciences/niceopdivoappraisal.pdf)

14 Wang L, Wang H, Chen H, Wang WD, Chen XQ, Geng QR, Xia ZJ \& Lu Y. Serum levels of soluble programmed death ligand 1 predict treatment response and progression free survival in multiple myeloma. Oncotarget 20156 41228-41236. (https://doi. org/10.18632/oncotarget.5682)

15 Rossille D, Gressier M, Damotte D, Maucort-Boulch D, Pangault C, Semana G, Le Gouill S, Haioun C, Tarte K, Lamy T, et al. High level of soluble programmed cell death ligand 1 in blood impacts overall survival in aggressive diffuse large B-cell lymphoma: results from a French multicenter clinical trial. Leukemia 201428 2367-2375. (https://doi.org/10.1038/leu.2014.137)

16 Fukuda T, Kamai T, Masuda A, Nukui A, Abe H, Arai K \& Yoshida K. Higher preoperative serum levels of PD-L1 and B7-H4 are associated with invasive and metastatic potential and predictable for poor response to VEGF-targeted therapy and unfavorable prognosis of renal cell carcinoma. Cancer Medicine 20165 1810-1820. (https://doi. org/10.1002/cam4.754)

17 Finkelmeier F, Canli Ö, Tal A, Pleli T, Trojan J, Schmidt M, Kronenberger B, Zeuzem S, Piiper A, Greten FR, et al. High levels of the soluble programmed death-ligand (sPD-L1) identify hepatocellular carcinoma patients with a poor prognosis. European Journal of Cancer 201659 152-159. (https://doi.org/10.1016/j. ejca.2016.03.002)

18 Okuma Y, Hosomi Y, Nakahara Y, Watanabe K, Sagawa Y \& Homma S. High plasma levels of soluble programmed cell death ligand 1 are prognostic for reduced survival in advanced lung cancer. Lung Cancer 2017104 1-6. (https://doi.org/10.1016/j. lungcan.2016.11.023)

19 Takahashi N, Iwasa S, Sasaki Y, Shoji H, Honma Y, Takashima A, Okita NT, Kato K, Hamaguchi T \& Yamada Y. Serum levels of soluble programmed cell death ligand 1 as a prognostic factor on the firstline treatment of metastatic or recurrent gastric cancer. Journal of Cancer Research and Clinical Oncology 2016142 1727-1738. (https:// doi.org/10.1007/s00432-016-2184-6)

20 Caixeiro NJ, Aghmesheh M, de Souza P \& Lee CS. The Centre for Oncology Education and Research Translation (CONCERT) biobank. Open Journal of Bioresources 20152 e3. (https://doi.org/10.5334/ojb.ai)

21 Centre for Oncology Education and Research Translation Biobank (CONCERT). Liverpool, NSW, Australia: Ingham Institute for Applied Medical Research. (available at: https://biobank. inghaminstitute.org.au/)

22 Kruger S, Legenstein ML, Rösgen V, Haas M, Modest DP, Westphalen CB, Ormanns S, Kirchner T, Heinemann V, Holdenrieder S, et al. Serum levels of soluble programmed death protein 1 (sPD-1) and soluble programmed death ligand 1 (sPD-L1) in advanced pancreatic cancer. Oncoimmunology 20176 e1310358. (https://doi.org/10.1080/2162402X.2017.1310358)

23 Kim HJ, Park S, Kim KJ \& Seong J. Clinical significance of soluble programmed cell death ligand-1 (sPD-L1) in hepatocellular carcinoma patients treated with radiotherapy. Radiotherapy and Oncology 2018129 130-135. (https://doi.org/10.1016/j. radonc.2017.11.027)

24 Nagato T, Ohkuri T, Ohara K, Hirata Y, Kishibe K, Komabayashi Y, Ueda S, Takahara M, Kumai T, Ishibashi K, et al. Programmed deathligand 1 and its soluble form are highly expressed in nasal natural killer/T-cell lymphoma: a potential rationale for immunotherapy. Cancer Immunology, Immunotherapy 201766 877-890. (https://doi. org/10.1007/s00262-017-1987-x)

25 Patel SP \& Kurzrock R. PD-L1 expression as a predictive biomarker in cancer immunotherapy. Molecular Cancer Therapeutics 201514 847-856. (https://doi.org/10.1158/1535-7163.MCT-14-0983)

26 Ha H, Nam AR, Bang JH, Park JE, Kim TY, Lee KH, Han SW, Im SA, Kim TY, Bang YJ, et al. Soluble programmed death-ligand 1 (sPDL1) and neutrophil-to-lymphocyte ratio (NLR) predicts survival in advanced biliary tract cancer patients treated with palliative chemotherapy. Oncotarget 20167 76604-76612. (https://doi. org/10.18632/oncotarget.12810)

27 Sorensen SF, Demuth C, Weber B, Sorensen BS \& Meldgaard P. Increase in soluble PD-1 is associated with prolonged survival in patients with advanced EGFR-mutated non-small cell lung cancer treated with erlotinib. Lung Cancer 2016100 77-84. (https://doi. org/10.1016/j.lungcan.2016.08.001)

28 Zhao J, Zhang P, Wang J, Xi Q, Zhao X, Ji M \& Hu G. Plasma levels of soluble programmed death ligand-1 may be associated with overall survival in nonsmall cell lung cancer patients receiving thoracic radiotherapy. Medicine 201796 e6102. (https://doi.org/10.1097/ MD.0000000000006102)

29 Zhou J, Mahoney KM, Giobbie-Hurder A, Zhao F, Lee S, Liao X, Rodig S, Li J, Wu X, Butterfield LH, et al. Soluble PD-L1 as a biomarker in malignant melanoma treated with checkpoint blockade. Cancer Immunology Research 20175 480-492. (https://doi. org/10.1158/2326-6066.CIR-16-0329)

30 Boffa DJ, Graf RP, Salazar MC, Hoag J, Lu D, Krupa R, Louw J, Dugan L, Wang Y, Landers M, et al. Cellular expression of PD-L1 in the peripheral blood of lung cancer patients is associated with worse survival. Cancer Epidemiology, Biomarkers and Prevention 201726 1139-1145. (https://doi.org/10.1158/1055-9965.EPI-17-0120)

31 Zhang Y, Zhu W, Zhang X, Qu Q \& Zhang L. Expression and clinical significance of programmed death-1 on lymphocytes and programmed death ligand-1 on monocytes in the peripheral blood of patients with cervical cancer. Oncology Letters 201714 7225-7231. (https://doi.org/10.3892/ol.2017.7105)

32 Zheng Z, Bu Z, Liu X, Zhang L, Li Z, Wu A, Wu X, Cheng X, Xing X, Du $\mathrm{H}$, et al. Level of circulating PD-L1 expression in patients with advanced gastric cancer and its clinical implications. Chinese Journal of Cancer Research 201426 104-111. (https://doi.org/10.3978/j. issn.1000-9604.2014.02.08)

33 Frigola X, Inman BA, Krco CJ, Liu X, Harrington SM, Bulur PA, Dietz AB, Dong H \& Kwon ED. Soluble B7-H1: differences in production between dendritic cells and T cells. Immunology Letters 2012142 78-82. (https://doi.org/10.1016/j.imlet.2011.11.001)

34 Davies LC, Heldring N, Kadri N \& Le Blanc K. Mesenchymal stromal cell secretion of programmed death-1 ligands regulates $\mathrm{T}$ cell mediated immunosuppression. Stem Cells 201735 766-776. (https:// doi.org/10.1002/stem.2509)

35 Alsaab HO, Sau S, Alzhrani R, Tatiparti K, Bhise K, Kashaw SK \& Iyer AK. PD-1 and PD-L1 checkpoint signaling inhibition for cancer immunotherapy: mechanism, combinations, and clinical outcome. Frontiers in Pharmacology 20178 561. (https://doi.org/10.3389/ fphar.2017.00561)

36 Gong J, Chehrazi-Raffle A, Reddi S \& Salgia R. Development of PD-1 and PD-L1 inhibitors as a form of cancer immunotherapy: a comprehensive review of registration trials and future considerations. Journal for ImmunoTherapy of Cancer 20186 8. (https://doi. org/10.1186/s40425-018-0316-z) 
37 Mehnert JM, VA BM, Aggarwal RR, Lin C-C, Prawira A, et al. Pembrolizumab for advanced papillary or follicular thyroid cancer: preliminary results from the phase 1b KEYNOTE-028 study. Journal of Clinical Oncology 201634 (15_Supplement) 6091. (https://doi. org/10.1200/JCO.2016.34.15_suppl.6091)

38 Cheng HY, Kang PJ, Chuang YH, Wang YH, Jan MC, Wu CF, Lin CL, Liu CJ, Liaw YF, Lin SM, et al. Circulating programmed death-1 as a marker for sustained high hepatitis B viral load and risk of hepatocellular carcinoma. PLoS ONE 20149 e95870. (https://doi. org/10.1371/journal.pone.0095870)

39 Yi J, Kim C \& Gelfand CA. Inhibition of intrinsic proteolytic activities moderates preanalytical variability and instability of human plasma. Journal of Proteome Research 20076 1768-1781. (https://doi.org/10.1021/pr060550h)

40 Ryan M, Huebinger GX, Wilhelmsen KC, Diaz-Arrastia R, Zhang F, O'Bryant SE \& Barber RC. Comparison of protein concentrations in serum versus plasma from Alzheimer's patients. Advances in Alzheimer's Disease 20121 51-58.

41 Yi M, Jiao D, Xu H, Liu Q, Zhao W, Han X \& Wu K. Biomarkers for predicting efficacy of PD-1/PD-L1 inhibitors. Molecular Cancer 2018 17 129-. (https://doi.org/10.1186/s12943-018-0864-3)
42 Udall M, Rizzo M, Kenny J, Doherty J, Dahm S, Robbins P \& Faulkner E. PD-L1 diagnostic tests: a systematic literature review of scoring algorithms and test-validation metrics. Diagnostic Pathology 201813 12-. (https://doi.org/10.1186/s13000018-0689-9)

43 Gaule P, Smithy JW, Toki M, Rehman J, Patell-Socha F, Cougot D, Collin P, Morrill P, Neumeister V \& Rimm DL. A quantitative comparison of antibodies to programmed cell death 1 ligand 1. JAMA Oncology 20173 256-259. (https://doi.org/10.1001/ jamaoncol.2016.3015)

44 Manson G, Norwood J, Marabelle A, Kohrt H \& Houot R. Biomarkers associated with checkpoint inhibitors. Annals of Oncology 201627 1199-1206. (https://doi.org/10.1093/annonc/mdw181)

45 Zhu X \& Lang J. Soluble PD-1 and PD-L1: predictive and prognostic significance in cancer. Oncotarget 20178 97671-97682. (https://doi. org/10.18632/oncotarget.18311)

46 Takeuchi M, Doi T, Obayashi K, Hirai A, Yoneda K, Tanaka F \& Iwai Y. Soluble PD-L1 with PD-1-binding capacity exists in the plasma of patients with non-small cell lung cancer. Immunology Letters 2018196 155-160. (https://doi.org/10.1016/j. imlet.2018.01.007

Received in final form 11 June 2019

Accepted 28 June 2019

Accepted Preprint published online 28 June 2019
This work is licensed under a Creative Commons Attribution-NonCommercial 4.0 International License. ded from Bioscientifica.com at $04 / 26 / 2023$ 12:15:41PM 